\title{
Towards the development of a cyber-physical measurement system (CPMS): case study of a bioinspired soft growing robot for remote measurement and monitoring applications
}

\author{
Stanislao Grazioso ${ }^{1}$, Annarita Tedesco ${ }^{2}$, Mario Selvaggio ${ }^{3}$, Stefano Debei $^{4}$, Sebastiano Chiodini ${ }^{4}$ \\ ${ }^{1}$ Department of Industrial Engineering, University of Naples Federico II, Naples, Italy \\ 2 IMS Laboratory, University of Bordeaux, Bordeaux, France \\ ${ }^{3}$ Department of Electrical Engineering and Information Technology, University of Naples Federico II, Naples, Italy \\ ${ }^{4}$ Department of Industrial Engineering, University of Padova, Padova, Italy
}

\begin{abstract}
The most effective expression of the 4.0 Era is represented by cyber-physical systems (CPSs). Historically, measurement and monitoring systems (MMSs) have been an essential part of CPSs; however, by introducing the 4.0 enabling technologies into MMSs, a MMS can evolve into a cyber-physical measurement system (CPMS). Starting from this consideration, this work reports a preliminary case study of a CPMS, namely an innovative bioinspired robotic platform that can be used for measurement and monitoring applications in confined and constrained environments. The innovative system is a "soft growing" robot that can access a remote site through controlled lengthening and steering of its body via a pneumatic actuation mechanism. The system can be endowed with different sensors at the tip, or along its body, to enable remote measurement and monitoring tasks; as a result, the robot can be employed to effectively deploy sensors in remote locations. In this work, a digital twin of the system is developed for simulation of a practical measurement scenario. The ultimate goal is to achieve a self-adapting, fully/partially autonomous system for remote monitoring operations to be used reliably and safely for the inspection of unknown and/or constrained environments.
\end{abstract}

\section{Section: RESEARCH PAPER}

Keywords: 4.0 Era; soft growing robots; remote monitoring; monitoring systems; remote sensing

Citation: Stanislao Grazioso, Annarita Tedesco, Mario Selvaggio, Stefano Debei, Sebastiano Chiodini, Towards the development of a cyber-physical measurement system (CPMS): case study of a bioinspired soft growing robot for remote measurement and monitoring applications, Acta IMEKO, vol. 10, no. 2 , article 15, June 2021, identifier: IMEKO-ACTA-10 (2021)-02-15

Section Editor: Francesco Lamonaca, University of Calabria, Italy

Received May 4, 2021; In final form May 11, 2021; Published June 2021

Copyright: This is an open-access article distributed under the terms of the Creative Commons Attribution 3.0 License, which permits unrestricted use, distribution, and reproduction in any medium, provided the original author and source are credited.

Corresponding author: Stanislao Grazioso, e-mail: stanislao.grazioso@unina.it

\section{INTRODUCTION}

The 4.0 Era is characterized by an innovative multidisciplinary approach which addresses technical challenges by seeking transverse solutions to both technological and methodological problems [1]-[8]. The most effective expression of the 4.0 paradigm is represented by cyber-physical systems (CPSs), i.e., smart systems that include engineered interacting networks of physical and computational components, able to monitor and control the physical environment [9]-[14]. From this definition, it appears that measurement and monitoring systems (MMSs) are essential for the implementation of CPSs. However, MMSs are generally considered as subordinate elements of a CPS: they provide source of information for the CPS (i.e., connection to the physical world) but do not participate in any higher-level actions of the CPS (i.e., conversion, cyber, cognition, configuration) [15]. MMSs are historically seen as responsible for sensing the conditions from the physical environment, rather than as providers of higher-level intelligent information.

However, the suitable adoption of the enabling technologies can reshape the role of MMSs in the 4.0 Era. This requires a fundamental change of perspective, in which the enabling technologies stop being employed as external superstructures for MMSs, and become embedded solutions, intrinsically present in the architecture of the MMS, and fully effective through an adequate metrological configuration. This approach emphasizes the holistic nature of monitoring and paves the way for 4.0 transition-driven monitoring systems. Through the wise adoption of the 4.0 enabling technologies, MMSs can turn into self-aware, 
self-conscious, self-maintained entities, able to generate highly valued insights, just like CPSs. This means that MMSs can evolve into cyber-physical measurement systems (CPMSs), thus becoming a proactive expression of the $4.0 \mathrm{Era}$, strengthening not only the role of measurement, but the performance of the overall 4.0 ecosystem.

Starting from these considerations, this work introduces a definition for the CPMSs and presents a preliminary case study, i.e., a "soft growing" robot for measurement and monitoring applications in constrained and confined remote environments. This system is a concrete example of CPMS for its ability to sense itself within the environment (i.e., self-awareness), to adapt itself to the surrounding environment thanks to its softness and growth and steering capabilities (i.e., self-configure), and to autonomously predict navigation path towards the inspection target (i.e., selfpredict $)$. Embedding multiple 4.0 enabling technologies in one measurement and monitoring system represents a promising solution for achieving the CPMS capabilities.

This work is an extension of our previous conference paper [16], where the general idea was preliminarily introduced. This paper is organized as follows. In Section 2, the state-of-the-art of robotic technologies is presented, with focus on measurement and monitoring remote applications in difficult-to-reach environments. Then, in Section 3, the definition for the CPMSs is introduced. Section 4 relates to the design and implementation of the soft growing robot and shows a simulated measurement and monitoring scenario of the CPMS in a remote location. Finally, in Section 6, conclusions are drawn, and the future work is outlined.

\section{BACKGROUND}

As well known, robotic technologies are largely used for carrying out remote measurement tasks, especially in environments that are hazardous, dangerous or difficult to reach for humans [17], [18]. Nevertheless, there are several applications in which traditional rigid-bodied robot technologies cannot be used. This occurs, for example, when measurements must be carried out in confined, constrained, or unknown environments (e.g., inspection of difficult-to-reach industrial environments, exploration of archaeological sites which are often inaccessible and fragile).

One technological solution suitable to perform this kind of tasks is represented by soft continuum robots [19], i.e. robots with a continuously deformable mechanical structure, whose design is inspired from the principles of shaping, movements, sensing and control of soft biological systems [20]. In the literature, there are several examples of soft continuum robots for remote measurement applications, in different fields: space, airlines, nuclear, marine (inspection and maintenance), medical (minimally invasive surgery), and so on [21].

One limitation of soft continuum robots is their limited workspace, as they usually have a fixed base and a pre-established length; this can be a problem for tasks that require inspection and exploration of large environments. To overcome this issue, soft continuum robots can be endowed with locomotion capabilities, by using tethered/untethered fluidic or cable-driven actuators, taking inspiration from the animal movements (snake, earthworms, caterpillars) [22]-[24]. However, this solution involves a relative movement between the robot and the environment, which can lead to low energy efficiency of the robot when high sliding friction is present.
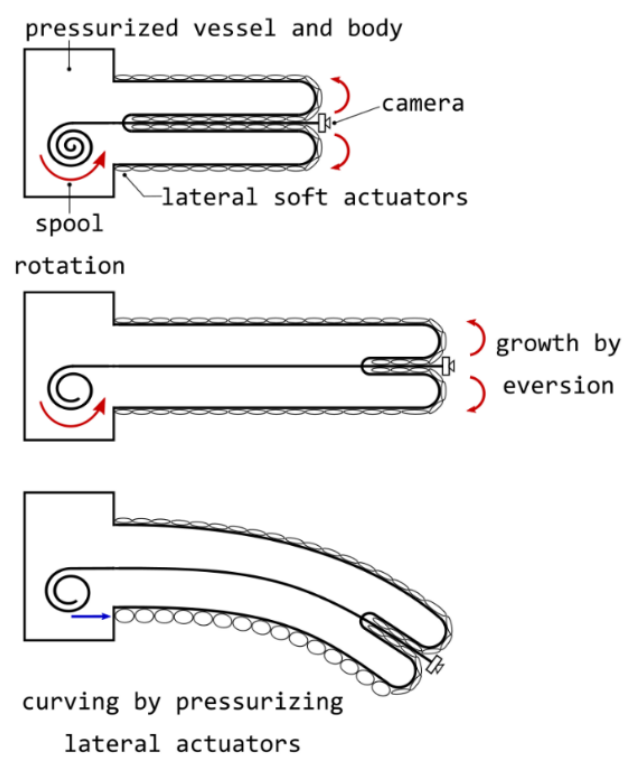

Figure 1. Example of the concept of a soft growing robot that achieves enhanced mobility through growth (top image). Concept of growth by eversion of material rolled onto a spool (central image); and the concept of curving by pressurization, with contraction (bottom image) of soft actuators placed and sealed on the main body. In the example, a camera is placed on the tip of the robot.

A recent design solution of soft continuum robots achieves enhanced mobility through growth rather than locomotion, taking inspiration from the growing process of plants and vines [25]. These robots, referred to as soft growing robots, achieve mobility by everting new material at the tip: this enables lengthening without relative movements between the robot's body and the environment. With soft growing robots, the inspection and exploration length of remote environments is therefore limited only by the amount of robot's body material that can be transported on the field. Although different mechanisms have been used to enable this form of apical extension [26], recently, pneumatically-driven solutions have achieved great potentials [27]. In these systems, the growing process is implemented by pressurizing a fluid (typically, air) inside a chamber created by a self-folded cylindrical body, which unfolds by everting new material from the tip; this enables the forward growth of the robot's body through lengthening. While growing, the robot's body can be curved/steered by additional actuators distributed along its body (e.g., pneumatic artificial muscles [28], artificial tendons, etc.). The contraction of these additional actuators on one side causes bending (or kinking) along that direction. An example of the growing and steering processes of soft growing robots is shown in Figure 1.

\section{CYBER-PHYSICAL MEASUREMENT SYSTEM}

The concept of CPMS is built on the 5C architecture [10] used for CPS, as shown in Figure 2. It consists of the following five levels:

1. Connection layer: connected with the physical world where the measurements are collected, and the sensing is performed.

2. Conversion layer: responsible for very first processing for endowing the system with self-awareness capabilities (i.e., reconstruction of its internal state).

3. Cyber layer: responsible for the development of the digital twin model of the measurement system and for 


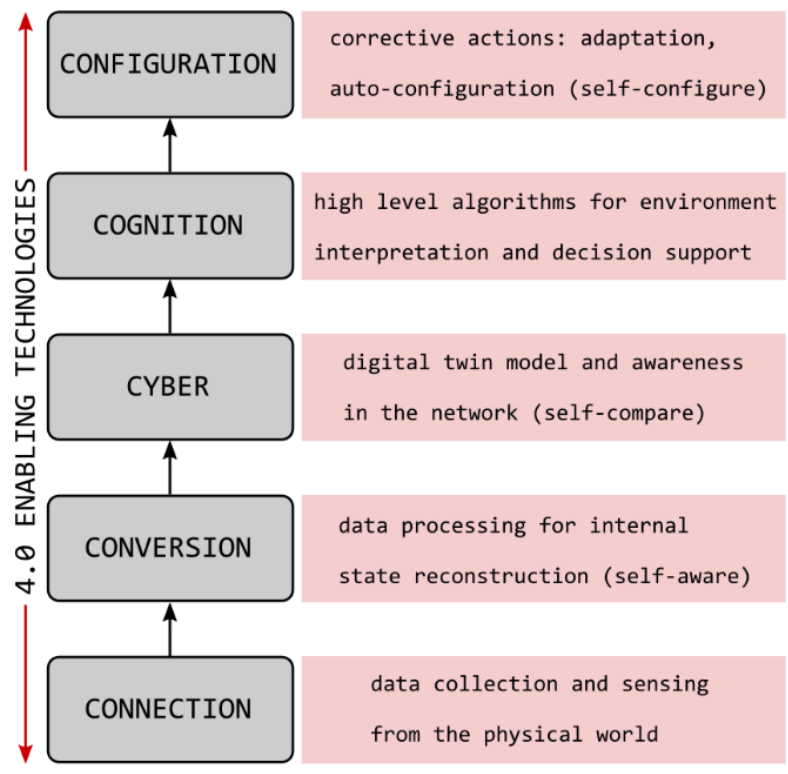

Figure 2. Description of a CPMS according to the $5 \mathrm{C}$ architecture.

endowing the system with self-comparison capabilities (i.e., self-awareness within the network).

4. Cognition layer: responsible for cognition and reasoning, i.e., high-level models and algorithms to endow the measurement system with decision support capabilities.

5. Configuration layer: starting from the knowledge generated by the cognitive level, this layer generates corrective actions, as adaptation, and reactiveness to the environments.

A CPMS can be defined as a novel form of MMS which, in addition to collecting data from the physical world, is able to provide higher-level information thanks to the use of suitable models and 4.0 enabling technologies. Similarly to a CPS, a CPMS has knowledge of its state in time and space (self-awareness) and with respect to other systems in the network (self-comparison); it is capable of enforcing actions for its own maintenance (selfmaintain), predicting its own evolution in time and space (selfpredict), and adapting to the environment (self-configure).
Drawing a comparison with the master-slave architecture, historically MMSs represented the slaves (mostly dedicated to data collection) of a master (i.e., the CPS itself). By evolving into CPMSs, instead, MMSs become CPSs among CPSs; eventually, the current master-slave relationship between MMSs and CPSs turns into a peer-to-peer cooperation.

\section{CASE STUDY: THE SOFT GROWING ROBOT}

This section addresses the design and implementation of the soft growing robot, as a preliminary example of CPMS to be used for applications within confined and constrained remote environments. First, the design requirements of the system are presented. Successively, its major components (i.e., the robotic platform and the electronic control unit) are described in detail. Finally, an example of a practical measurement scenario through a simulated digital twin of the system is presented. The architecture of the soft growing robot is shown in Figure 3.

\subsection{Requirements}

The requirements of the soft growing robot are the following:

- Access within environments with small cross sections (with minimum dimension equal to $100 \mathrm{~mm}$ );

- High inspection/exploration length (up to $10 \mathrm{~m}$ ) while maintaining portability;

- Controllable growth;

- Steering/curving capability; and

- Human situation awareness.

The long-term goal is to develop the first soft growing robot endowed with model-based strategies [29] for planning [30], control and navigation to accomplish remote measurement tasks. These requirements give the possibility to develop a generalpurpose platform for inspection and exploration usable in a wide range of scenarios.

\subsection{Robotic platform}

The robotic platform is mainly constituted by the robot base (where the body material is stored) and the robot body (which grows and accesses the remote environment). The CAD model of the robotic platform is shown in Figure 4.

The robot base is the container of the unfolded robot body and represents the pressurized vessel when the robot is in

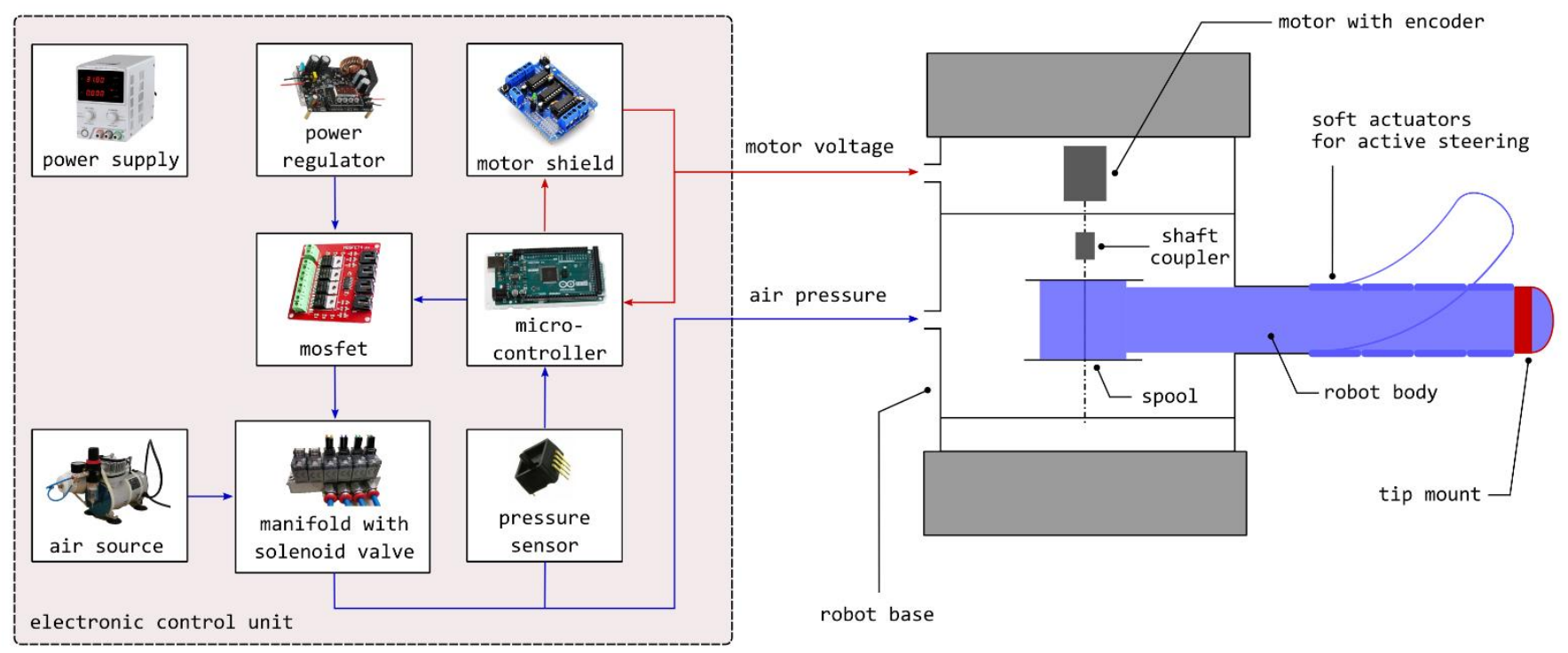

Figure 3. Architecture of the proposed soft growing robotic system (dimensions not in scale). 
operation. It is formed by an acrylic cylinder with two end caps (QC-108 Qwik cap, Fernco Inc., Davison, MI). The spool for rolling out the robot material is driven by a DC motor $(6408 \mathrm{~K} 27$, McMaster-Carr Inc., Douglasville, GA) with a magnetic encoder which allows growing/retracting the robot body and measuring its current length. The vessel of the robot base is a cylinder with diameter $d$ equal to $25 \mathrm{~cm}$ and height $b$ equal to $50 \mathrm{~cm}$.

The main body of the soft growing robot is made of an airtight tube which is flexible but not stretchable. During the eversion process, the material should slide relative to itself with negligible friction; and should guarantee major durability for field applications. To this end, a double side silicon-coated ripstop nylon (Rockywoods Fabric LLC, Loveland, CO) is used as a material for the main body of the robot. The soft robot body is rolled up and fixed from one hand around a spool inside the base vessel: when pressurized, the material is pushed outside the robot base through an opening and everts from the tip of the robot. When fully extended, the robot body achieves maximum dimensions corresponding to a diameter $p$ of $10 \mathrm{~cm}$ and a maximum length $l$ of $10 \mathrm{~m}$.

The forward growth is controllable by finding a suitable balance between the desired air pressure inside the vessel and the desired spool rotation, and thus, motor angular velocity about the axis of the spool. For guaranteeing a reversible steering/curving of the robot body, soft pneumatic actuators (made of the same material of the robot's body) are placed along the entire length of the robot [28]: the steering/curving control is guaranteed by a suitable pressurization of these additional actuators, considering models of curvature/deformations of the robot's shape [31]. During the retraction process of the robot's body, to avoid structural kinking, suitable mechanisms to drive the retraction should be foreseen [32].

\subsection{Electronic control unit}

The electronic control unit is composed by two sub-systems: one for generating the desired air pressure for pressurization of the vessel, and one for generating the desired voltage for the DC motor for growth/retraction of the robot body. The pneumatic circuit regulates the air pressure by pulse-width modulation (PWM), which involves the controlled timing of the opening and closing of solenoid valves (SY114-5LOU, SMC) through a mosfet board (based on IRF540 mosfets, STMicroelectronics), with pressure sensors (ASDXAVX100PGAA5, Honeywell) providing feedback. The pneumatic circuit is an essential component of the robot, as it is responsible for the growth process (one pneumatic tube for the main tube of the robot body) and the steering of the robot body (one pneumatic tube for each of the serial soft actuators placed along the robot body). This PWM-based pneumatic homemade circuit substitutes more expensive solutions based on professional closed-loop pressure regulators. The prototype of the developed electronic control unit (related to the compressed air circuit) is shown in Figure 5.

\subsection{Example of a practical measurement scenario}

The practical measurement scenario consists of a human operator performing visual inspection in a remote site through the proposed soft growing robot, endowed with a tip-mounted camera. An input device is used by the human operator to impart growing/steering commands.

A digital twin of the measurement system is built in V-REP including the model of the robot (modelled as a growing constant curvature robot [33]), the model of the cameras as well as the model of the environment. The constant curvature assumption is reasonable when artificial pneumatic muscles are used to steer the robot, as shown in [30]. Snapshots of the measurement scenario are shown in Figure 6, where we can see the remotely operated soft growing robot approaching and inspecting a target (red box) within the simulated remote site.

The soft growing robotic platform represents a novel technology for the inspection of confined and constrained remote environments that are not accessible by current technologies. Furthermore, it represents a suitable platform for enabling measurement applications in large, GPS-denied environments. In addition to industrial inspections, this robotic platform may be used for exploration purposes or even for search and rescue applications after accidents, earthquakes, and collapses of buildings. Finally, an additional application is the sensor delivery in difficult-to-reach remote sites.

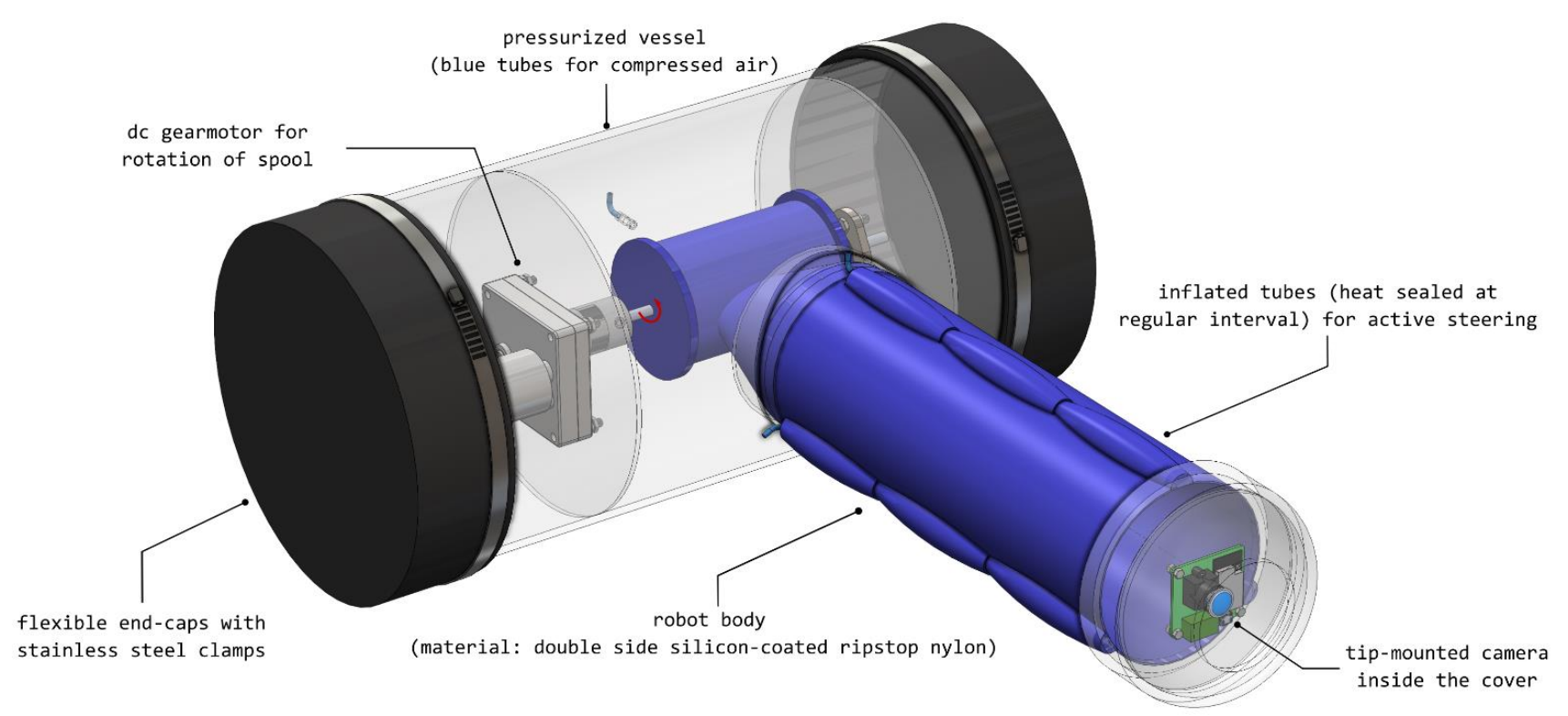

Figure 4. CAD representation of the robotic platform. 


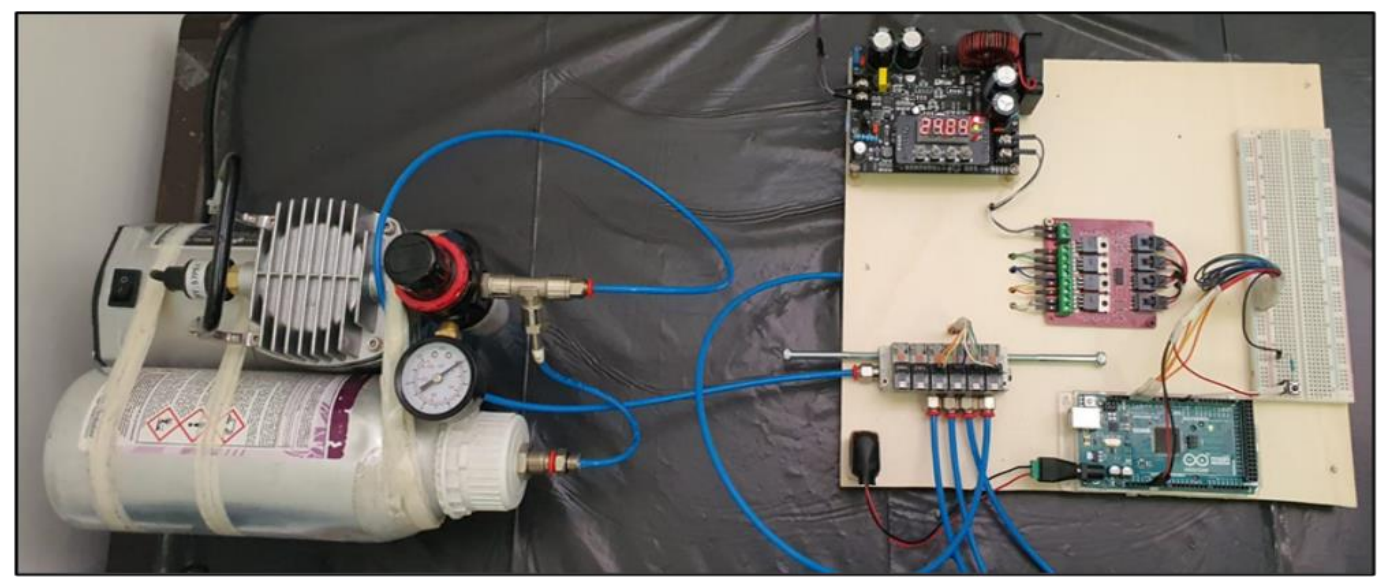

Figure 5. Picture of the electronic control unit (pneumatic board).

\section{CONCLUSION AND FUTURE WORK}

In this work, a definition for CPMS was introduced and a soft growing robot was presented as a case study. A CPMS can be considered as a 4.0-oriented evolution of traditional MMS; as a matter of fact, thanks to the adoption of 4.0 enabling technologies, a MMS is not only seen as a system for collecting data, but also for data processing and interpretation.

The soft growing robot as a CPMS is intended to be used for remote measurement and monitoring applications in constrained and confined environments. The proposed system consists of a robot, to be placed outside the remote site, and a soft body that accesses the site through growth, with the possibility of controlling length and steering. At the tip of the system, a sensor can be placed to enable remote measurement tasks, or a sensor can be deployed through the robot body when the target is reached. In this work, we have considered a tip-mounted camera in the system. The benefits of using soft growing robots for remote measurement and monitoring applications are access within small-scaled sections; high inspection/exploration length; transportability; controllable growth and steering; safe interaction with the environment; and ability to perform sensor deployment.

To get closer to a full definition of CPMS, future work will be dedicated to embedding additional 4.0 enabling technologies (e.g., artificial intelligence algorithms) in the CMPS, for endowing monitoring system with autonomous planning/navigation capabilities. Also, effort will be dedicated to motion analysis and control in highly constrained situations. Additionally, a set of practical applications cases will be identified, to experiment the system and assess its metrological performance. Finally, suitable sensing technologies and processing strategies will be developed to enhance the metrological performance of the system (e.g., in terms of resolution, reliability and accuracy of interaction with the environment). The ultimate goal is to achieve a self-adapting, fully autonomous system for remote monitoring operations to be used reliably and safely for the inspection of unknown and/or constrained and confined environments.

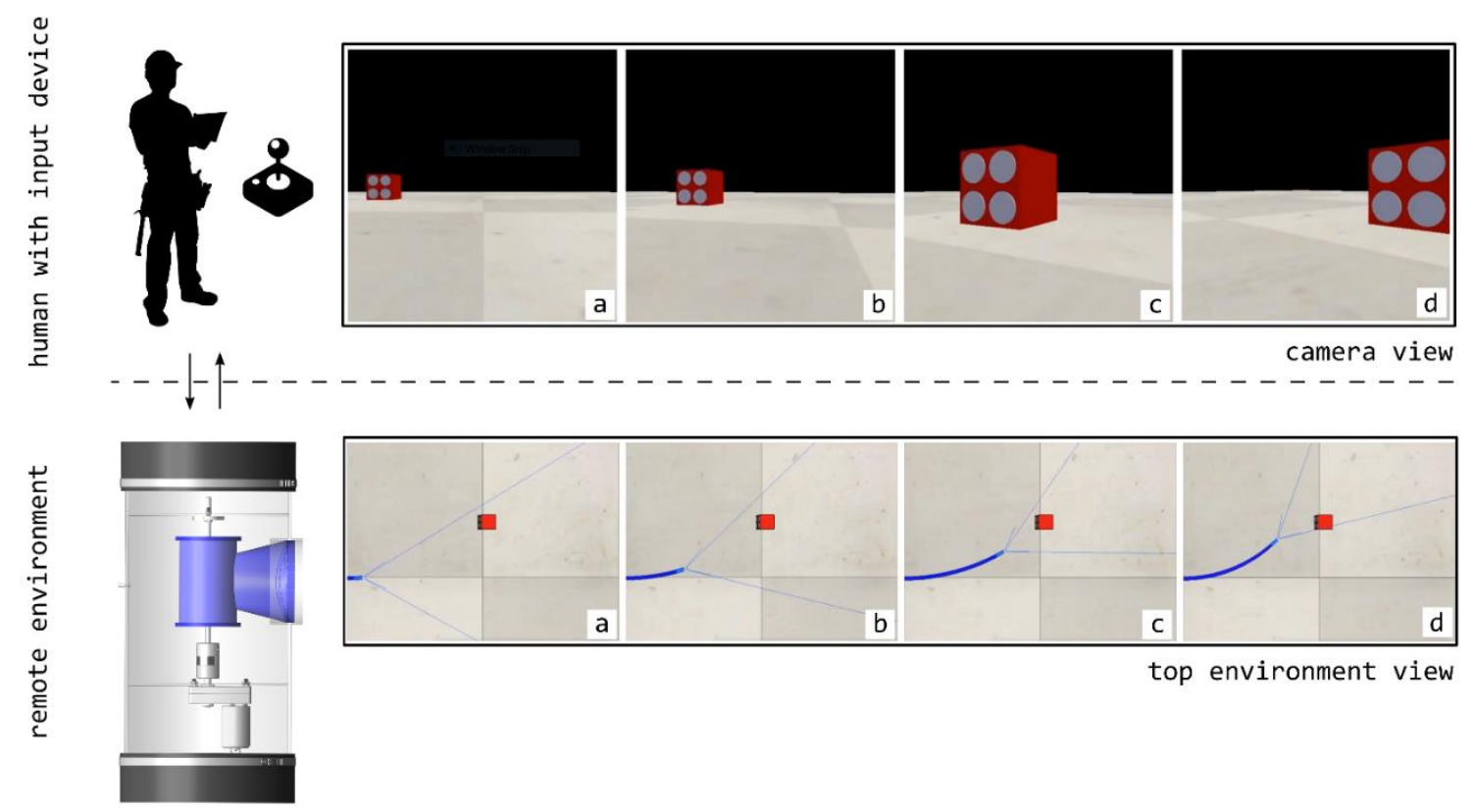

Figure 6. Example scenario for remote inspection through the proposed soft growing robot. By using a suitable input device, the human operator drives the growth and steering of the robotic system in the remote environment, accessed through an access section which is slightly larger than the diameter of the robot's body. The human operator sees the remote environment through the tip-mounted camera. 


\section{REFERENCES}

[1] D. Seneviratne, L. Ciani, M. Catelani, D. Galar, Smart maintenance and inspection of linear assets: An Industry 4.0 approach, Acta IMEKO 7(1) (2018), pp. 50-56.

DOI: $10.21014 /$ acta imeko.v7i1.519

[2] T. I. Erdei, Z. Molnár, N. C. Obinna, G. Husi, A novel design of an augmented reality based navigation system \& its industrial applications, Acta IMEKO 7(1) (2018), pp. 57-62.

DOI: $\underline{10.21014 / \text { acta imeko.v7i1.528 }}$

[3] P. Arpaia, E. De Benedetto, L. Duraccio, Design, implementation, and metrological characterization of a wearable, integrated ARBCI hands-free system for health 4.0 monitoring, Measurement 177 (2021) art. no. 109280.

DOI: $10.1016 /$ j.measurement.2021.109280

[4] P. Arpaia, E. De Benedetto, C. A. Dodaro, L. Duraccio, G. Servillo, Metrology-based design of a wearable augmented reality system for monitoring patient's vitals in real time, IEEE Sensors Journal 21(9) (2021), pp. 11176-11183.

DOI: $10.1109 /$ JSEN.2021.3059636

[5] L. Angrisani, U. Cesaro, M. D'Arco, O. Tamburis, Measurement applications in industry 4.0: The case of an IoT-oriented platform for remote programming of automatic test equipment, ACTA IMEKO 8(2) (2019), pp. 62-69.

DOI: $10.21014 /$ acta imeko.v8i2.643

[6] R. Schiavoni, G. Monti, E. Piuzzi, L., Tarricone, A. Tedesco, E. De Benedetto, A. Cataldo, Feasibility of a wearable reflectometric system for sensing skin hydration, Sensors 20(10) (2020), art. no. 2833.

DOI: $10.3390 / \mathrm{s} 20102833$

[7] M. Prist, A. Monteriù, E. Pallotta, P. Cicconi, A. Freddi, F. Giuggioloni, E. Caizer, C. Verdini, S. Longhi, Cyber-physical manufacturing systems: An architecture for sensor integration, production line simulation and cloud services, Acta IMEKO 9(4) (2020), pp. 39-52.

DOI: $10.21014 /$ acta imeko.v9i4.731

[8] A. Cataldo, E. De Benedetto, L. Angrisani, G. Cannazza, E. Piuzzi, A microwave measuring system for detecting and localizing anomalies in metallic pipelines, IEEE Transactions on Instrumentation and Measurement 70 (2021) art. no 8001711. DOI: $10.1109 /$ TIM.2020.3038491

[9] Cyber-Physical Systems Public Working Group, Framework for cyber-physical systems: Volume 1, overview, version 1.0, NIST Special Publication, 1500-201, 2017. Online [Accessed 09 June 2021] https://pages.nist.gov/cpspwg/

[10] A. Ahmadi, C. Cherifi, V. Cheutet, Y. Ouzrout, A review of CPS 5 components architecture for manufacturing based on standards, in Proc. of IEEE 2017 11th International Conference on Software, Knowledge, Information Management and Applications (SKIMA), Sri Lanka, 06-08 December 2017, pp. 1-6. DOI: $10.1109 /$ SKIMA.2017.8294091

[11] A. Tedesco, M. Gallo, A. Tufano, A preliminary discussion of measurement and networking issues in cyber physical systems for industrial manufacturing, in Proc. of 2017 IEEE International Workshop on Measurement and Networking, M\&N 2017, Naples, Italy, 27-29 Sept. 2017.

DOI: $10.1109 /$ IWMN.2017.8078384

[12] A. Drago, S. Marrone, N. Mazzocca, R. Nardone, A. Tedesco, V. Vittorini, A model-driven approach for vulnerability evaluation of modern physical protection systems, Software and Systems Modeling 18 (2019), pp. 523-556.

DOI: $\underline{10.1007 / \mathrm{s} 10270-016-0572-7}$

[13] A. Sforza, C. Sterle, P. D'Amore, A. Tedesco, F. De Cillis, R. Setola, Optimization models in a smart tool for the railway infrastructure protection, in: CRITIS 2013: Critical Information Infrastructures Security, Lecture Notes in Computer Science, 8328, Amsterdam, The Netherlands, 16-18 Sept. 2013, pp. 191196.

DOI: $10.1007 / 978-3-319-03964-0 \quad 17$
[14] P. D'Amore, A. Tedesco, Technologies for the implementation of a security system on rail transportation infrastructures, in Topics in Safety, Risk, Reliability and Quality 27 (2015), pp. 123-141. DOI: $10.1007 / 978-3-319-04426-2$ - 7

[15] D. Yin, X. Ming, X. Zhang, Understanding data-driven cyberphysical-social system (D-CPSS) using a 7C framework in social manufacturing context, Sensors 20(18) (2020), art. no 5319. DOI: $\underline{10.3390 / \mathrm{s} 20185319}$

[16] S. Grazioso, A. Tedesco, M. Selvaggio, S. Debei, S. Chiodini, E. De Benedetto, G. Di Gironimo, A. Lanzotti, Design of a soft growing robot as a practical example of cyber-physical measurement systems, in Proc. 2021 IEEE International Workshop on Metrology for Industry 4.0 and IoT, Rome, Italy, 79 June 2021.

[17] M. Friedrich, G. Dobie, C. Chan, S. Pierce, W. Galbraith, S. Marshall, G. Hayward, Miniature mobile sensor platforms for condition monitoring of structures, IEEE Sensors Journal 9(11) (2009), pp. 1439-1448. DOI: $10.1109 /$ JSEN.2009.2027405

[18] M. Y. Moemen, H. Elghamrawy, S. N. Givigi, A. Noureldin, 3-d reconstruction and measurement system based on multi mobile robot machine vision, IEEE Transactions on Instrumentation and Measurement 70 (2021), pp. 1-9.

DOI: $10.1109 /$ TIM.2020.3026719

[19] C. Della Santina, M. G. Catalano, A. Bicchi, Soft robots, Berlin, Heidelberg: Springer, 2020. DOI: https://doi.org/10.1007/978-3-642-41610-1 146-2

[20] S. Kim, C. Laschi, B. Trimmer, Soft robotics: a bio inspired evolution in robotics, Trends in biotechnology 31(5) (2013), pp. 287-294.

DOI:10.1016/j.tibtech.2013.03.002

[21] L. Angrisani, S. Grazioso, G. Di Gironimo, D. Panariello, A. Tedesco, On the use of soft continuum robots for remote measurement tasks in constrained environments: a brief overview of applications, in Proc. of 2019 IEEE International Symposium on Measurements \&Networking (M\&N). IEEE, Catania, Italy, 810 July 2019, pp. $1-5$. DOI: $10.1109 /$ IWMN.2019.8805050

[22] W. Hu, G. Z. Lum, M. Mastrangeli, M. Sitti, Small-scale softbodied robot with multimodal locomotion, Nature 554 (2018), pp. 81-85. DOI: $10.1038 /$ nature25443

[23] I. H. Han, H. Yi, C.-W. Song, H. E. Jeong, S.-Y. Lee, A miniaturized wall-climbing segment robot inspired by caterpillar locomotion, Bioinspiration \& Biomimetics 12(4) (2017), 13 pages. DOI: $\underline{10.1088 / 1748-3190 / \mathrm{aa} 728 \mathrm{c}}$

[24] G. Gu, J. Zou, R. Zhao, X. Zhao, X. Zhu, Soft wall-climbing robots, Science Robotics 3(25) (2018), 12 pages. DOI: https://doi.org/10.1126/scirobotics.aat2874

[25] E. W. Hawkes, L. H. Blumenschein, J. D. Greer, and A. M. Okamura, A soft robot that navigates its environment through growth, Science Robotics 2(8) (2017), 8 pages. DOI: https://doi.org/10.1126/scirobotics.aan3028

[26] A. Sadeghi, A. Mondini, B. Mazzolai, Toward self-growing soft robots inspired by plant roots and based on additive manufacturing technologies, Soft robotics 4(3) (2017), pp. 211 223. DOI: https://doi.org/10.1089/soro.2016.0080

[27] J. D. Greer, T. K. Morimoto, A. M. Okamura, E. W. Hawkes, A soft, steerable continuum robot that grows via tip extension, Soft robotics 6 (2019), pp. 95-108. DOI: https://doi.org/10.1089/soro.2018.0034

[28] N. D. Naclerio, E. W. Hawkes, Simple, low-hysteresis, foldable, fabric pneumatic artificial muscle, IEEE Robotics and Automation Letters 5(2) (2020), pp. 3406-3413. DOI: $10.1109 /$ LRA.2020.2976309

[29] S. Grazioso, G. Di Gironimo, B. Siciliano, A geometrically exact model for soft continuum robots: The finite element deformation space formulation, Soft robotics 6 (2019), pp. 790-811. DOI: https://doi.org/10.1089/soro.2018.0047 
[30] M. Selvaggio, L. Ramirez, N. Naclerio, B. Siciliano, E. Hawkes, An obstacle-interaction planning method for navigation of actuated vine robots, in Proc. of 2020 IEEE International Conference on Robotics and Automation (ICRA), Paris, France, May 31- Aug. 31, 2020, pp. 3227-3233.

DOI: $10.1109 /$ ICRA40945.2020.9196587

[31] S. Grazioso, G. Di Gironimo, B. Siciliano, From differential geometry of curves to helical kinematics of continuum robots using exponential mapping, in Proc. of International Symposium on Advances in Robot Kinematics, Bologna, Italy, 01-05 July 2018, pp. 319-326.

DOI: $10.1007 / 978-3-319-93188-3 \quad 37$
[32] M. M. Coad, R. P. Thomasson, L. H. Blumenschein, N. S. Usevitch, E. W. Hawkes, A. M. Okamura, Retraction of soft growing robots without buckling, IEEE Robotics and Automation Letters 5(2) (2020), pp. 2115-2122. DOI: $10.1109 /$ LRA.2020.2970629

[33] S. Grazioso, G. Di Gironimo, B. Siciliano, Analytic solutions for the static equilibrium configurations of externally loaded cantilever soft robotic arms, in Proc. of 2018 IEEE International Conference on Soft Robotics(RoboSoft), Livorno, Italy, 24-28 April 2018, pp. 140-145.

DOI: 10.1109/ROBOSOFT.2018.8404910 